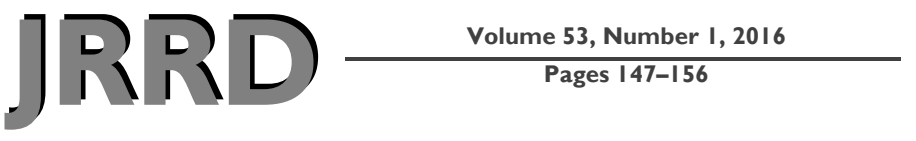

\title{
Implementation of telementoring for pain management in Veterans Health Administration: Spatial analysis
}

\author{
Evan P. Carey, MS; ${ }^{1-2 *}$ Joseph W. Frank, MD, MPH; ${ }^{1,3}$ Robert D. Kerns, PhD; ${ }^{4}$ P. Michael Ho, MD, PhD; ${ }^{1,5}$ \\ Susan R. Kirsh, MD, MPH ${ }^{6}$ \\ ${ }^{1}$ Department of Veterans Affairs (VA) Eastern Colorado Health Care System, Denver, CO; ${ }^{2}$ Department of Biostatistics \\ and Informatics, Colorado School of Public Health, Aurora, $\mathrm{CO} ;{ }^{3}$ Division of General Internal Medicine, University of \\ Colorado School of Medicine, Aurora, CO; ${ }^{4}$ VA Connecticut Healthcare System, West Haven, CT; and Yale School of \\ Medicine, New Haven, CT; ${ }^{5}$ Department of Medicine, University of Colorado School of Medicine, Aurora, CO; ${ }^{6}$ Louis \\ Stokes Cleveland VA Medical Center, Cleveland, OH; Department of Medicine, School of Medicine, Case Western \\ Reserve University, Cleveland, OH; and Office of Specialty Care, Veterans Health Administration, Washington, DC
}

\begin{abstract}
In 2011, the Veterans Health Administration (VHA) implemented a pilot telementoring program across seven healthcare networks called the Specialty Care Access Network-Extension for Community Healthcare Outcomes (SCAN-ECHO) for pain management. A VHA healthcare network is a group of hospitals and clinics administratively linked in a geographic area. We created a series of county-level maps in one network displaying (1) the location of Veterans with chronic pain, (2) VHA sites (i.e., coordinating center, other medical centers, outpatient clinics), (3) proportion of Veterans being seen in-person at pain specialty clinics, and (4) proportion of Veterans with access to a primary care provider participating in Pain SCAN-ECHO. We calculated the geodesic distance from Veterans' homes to nearest VHA pain specialty care clinics. We used logistic regression to determine the association between distance and Pain SCAN-ECHO primary care provider participation. Mapping showed counties closer to the Pain SCAN-ECHO coordinating center had a higher rate of Veterans whose providers participated in Pain SCAN-ECHO than those further away. Regression models within networks revealed wide heterogeneity in the reach of Pain SCAN-ECHO to Veterans with low spatial access to pain care. Using geographic information systems can reveal the spatial reach of technology-based healthcare programs and inform future expansion.
\end{abstract}

Key words: chronic pain, distance, ECHO, geographic information system, GIS, healthcare access, spatial, telemedicine, telementoring, Veteran, Veterans Health Administration.

\section{INTRODUCTION}

Technology-based healthcare programs designed to train primary care providers in the management of complex chronic conditions are being increasingly implemented [1-2]. The Project for the Extension for Community Healthcare Outcomes (Project ECHO) is one such telementoring program that initially focused on hepatitis $\mathrm{C}$ and has since expanded to other specialties, including chronic pain [3]. Past evaluations of Project ECHO (and other telementoring) initiatives have focused on comparative effectiveness of technology-based healthcare [4], assessment of intermediate patient outcomes [5],

\footnotetext{
Abbreviations: $\mathrm{CI}=$ confidence interval, GIS = geographic information systems, NRS = numeric rating scale, OR = odds ratio, Project ECHO = Project for the Extension for Community Healthcare Outcomes, SCAN-ECHO = Specialty Care Access Network-Extension for Community Healthcare Outcomes, VA = Department of Veterans Affairs, VHA = Veterans Health Administration.

*Address all correspondence to Evan P. Carey, MS; 13611 E. Colfax Ave, (151) A3-346D, Aurora, CO 80045. Email: Evan.Carey@va.gov http://dx.doi.org/10.1682/JRRD.2014.10.0247
} 
descriptions of programmatic scope [6], and qualitative data from providers' perspectives on barriers and facilitators [7]. A major goal of these initiatives is to overcome barriers to accessing specialty care. Specifically, distance from the nearest specialty care provider has been shown to be a barrier in multiple studies. However, prior spatial evaluations of technology-based healthcare programs have been limited to summaries of miles saved and maps of participating clinics [8-9].

Up to 50 percent of Veterans have one or more chronic pain conditions [10]. Further, up to 36 percent of Veterans live in rural areas [11]. These rural Veterans face geographic barriers to pain specialty care as Veterans Health Administration (VHA) specialty pain care clinics are typically located in urban medical centers. Starting in 2011, the VHA began a program based on Project ECHO called the Specialty Care Access Network-Extension for Community Healthcare Outcomes (SCAN-ECHO). The SCAN-ECHO program included a focus on chronic pain and sought to improve chronic pain care by extending specialty care expertise to primary care providers caring for Veterans who live far away from medical centers with pain specialists. To our knowledge, previous studies have not examined the spatial penetration of a specialty-specific telementoring program, contextualized by the underlying location of the target patient population and spatial barriers to specialty care.

The objective of this study was to evaluate the spatial reach of the VHA's Pain SCAN-ECHO program, contextualized by the spatial distribution of Veterans with chronic pain and in-person specialty pain care in seven VHA healthcare networks. Specifically, within one sample healthcare network with a Pain SCAN-ECHO program, we used geographic information systems (GIS) to map (1) the location of Veterans with chronic pain, (2) VHA sites (i.e., coordinating center, other medical centers, outpatient clinics), (3) proportion of Veterans being seen in-person at pain specialty clinics, and (4) proportion of Veterans with access to a primary care provider participating in Pain SCAN-ECHO. Using distance from Veterans' homes to nearest pain specialty care as a marker of spatial access, we used logistic regression models at the patient level to describe the association between access to specialty pain care and SCAN-ECHO primary care provider participation. We used similar logistic regression models to describe the association between access to pain care and in-person utilization as a comparison. Consistent with the goals of the Pain SCAN-
ECHO program, we hypothesized that greater distance from pain specialty care would be associated with increased primary care provider participation.

\section{METHODS}

\section{Population and Setting}

We identified all Veterans in seven VHA networks with chronic pain, defined as reporting at least one pain numeric rating score (NRS) $\geq 4$ during any inpatient or outpatient encounter in at least 3 distinct calendar mo during any 12 mo period from April 1, 2010, to December 31, 2013 ( $n=410,780$ ) (Figure 1). This definition of chronic pain is consistent with prior literature [12]. During inpatient and outpatient encounters, VHA patients report current pain using the NRS, with 0 equivalent to no pain and 10 equivalent to the worst possible pain [13]. We identified all primary care encounters starting $60 \mathrm{~d}$ prior to the first reported pain intensity rating. Veterans were linked to a primary care provider if they had at least three visits with the same provider. All Veterans with chronic pain in this study were successfully linked to primary care providers. Patients linked to primary care providers outside of the seven networks were excluded ( $n=$ $22,323)$. We excluded patients whose home address was missing $(n=8,420)$. We used GIS to determine whether a Veteran's home address was within the spatial boundaries of one of the seven healthcare networks. We included border counties of these networks. We excluded Veterans whose home address was not within those boundaries $(n=8,391)$.

\section{Pain SCAN-ECHO Program}

In 2011, the VHA implemented the Pain SCAN-ECHO program in 7 out of the 21 VHA healthcare networks. The VHA SCAN-ECHO program has been previously described [6]. Briefly, the SCAN-ECHO program uses communication technology such as video-conferencing to facilitate case-based mentoring between specialty care providers at coordinating centers and primary care providers at remote clinics. SCAN-ECHO participation is voluntary, and participating primary care providers receive continuing medical education credit. Primary care providers who presented patient cases at at least one Pain SCAN-ECHO sessions were considered exposed to the Pain SCANECHO program on the date of their first case presentation. Participation was validated by coordinating centers. 


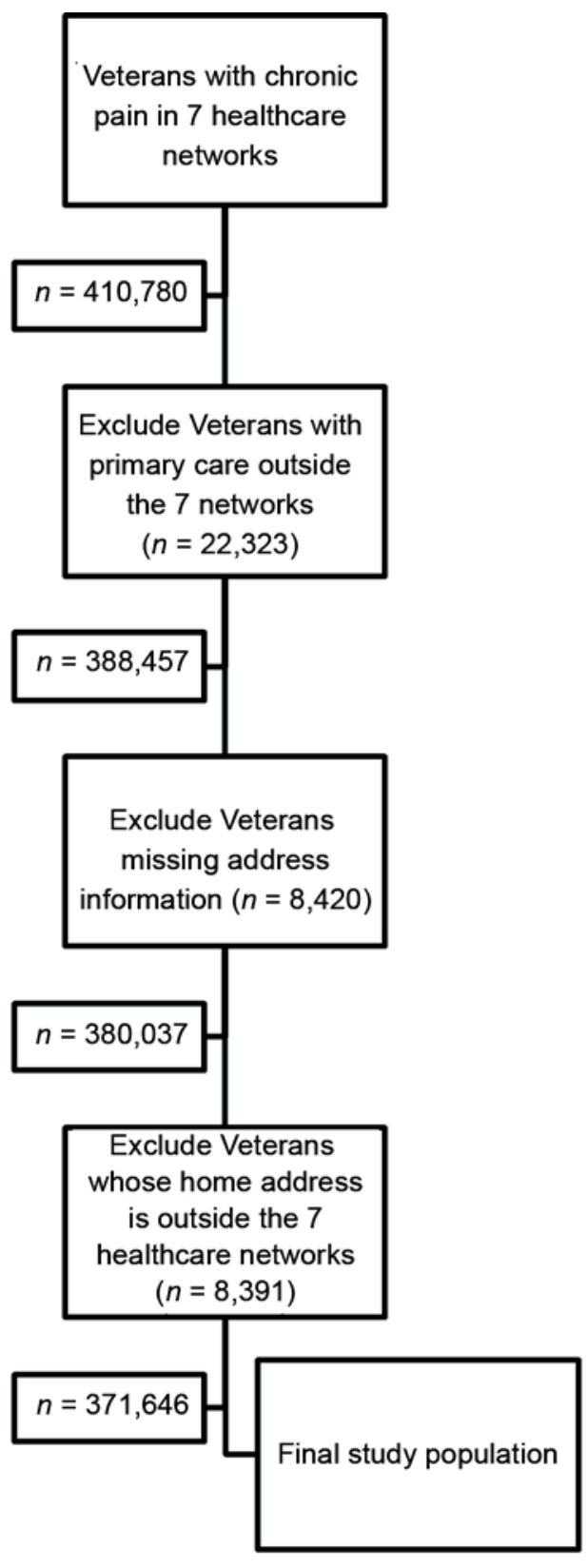

Figure 1.

Description of cohort exclusion criteria.

Patients were considered exposed to Pain SCAN-ECHO on the date that their primary care provider presented his or her first Pain SCAN-ECHO session.

\section{Pain Clinic Identification}

Specialty pain clinics are not available at every VHA medical center and clinic. We created a binary variable for whether pain specialty care was available at medical centers or clinics based on the observed number of nontelemedicine specialty pain clinic outpatient encounters of this chronic pain cohort. Specialty pain care is primarily available at medical centers. However, some community clinics offer specialty pain care. We contacted 15 sites with a borderline number of visits directly to assess specialty pain care availability, which informed our definition of specialty pain care availability. Medical centers with $\geq 100$ nontelemedicine visits coded with a pain clinic stop code were considered specialty pain care sites. Outpatient clinics with $\geq 200$ nontelemedicine visits coded with a pain clinic stop code were considered specialty pain care sites. Outpatient records, including site identifiers, were used to determine whether a Veteran had an inperson encounter in specialty pain clinic (i.e., not coded as telemedicine) in one of the sites with specialty pain care. Veterans meeting these criteria were considered to have received in-person specialty pain care.

\section{Geographic Information Systems}

Geographic files of the seven VHA networks and geocoded facility locations and designations were obtained from Department of Veterans Affairs (VA) Planning Systems and Support Group. United States' county, state, and nation boundaries were obtained from the U.S. Census Bureau. Veterans were aggregated to counties and health networks using GIS to determine whether a home address was within a county or health-network border. We calculated the geodesic ("as the crow flies") distance in miles from every Veteran's home to the nearest VHA facility designated a specialty pain care facility. This distance was used as a surrogate for access to pain care. We created a series of maps in a sample VHA network to display the geographic distribution of (1) the underlying distribution of Veterans with chronic pain, (2) the distribution of Veterans with access to a primary care provider participating in Pain SCAN ECHO, and (3) the distribution of Veterans seen in-person by specialty pain care.

\section{Statistical Analyses}

Each Veteran's rurality status was based on previously described classifications of urban, rural, and highly rural [14]. Highly rural Veterans were collapsed with rural Veterans to obtain a binary patient-level variable of urban or rural. Patient-level traits were aggregated to networks to establish the total number of Veterans with chronic pain, patients exposed to SCAN-ECHO, and patients who received in-person specialty pain care in each of the 
seven networks. Within each network and overall, the distribution of the distance from Veterans' homes to the nearest specialty pain care site was summarized as a median and interquartile range. We also calculated the proportion of patients living within 50 miles of a specialty pain care site.

We used logistic regression to determine the association between (1) distance and pain specialty care and (2) distance and Pain SCAN-ECHO primary care provider participation. Both models were fit for the entire population and for each network individually and summarized as odds ratios (ORs) with 95 percent confidence intervals (CIs) based on profiled likelihoods. The goal of these models was to quantify and contrast the overall relationships between the outcomes (SCAN-ECHO provider participation and in-person pain care) and distance. Accordingly, these models were not adjusted for other covariates. Preliminary data cleaning was done using SAS, version 9.3 (SAS Institute Inc; Cary, North Carolina). All logistic regression, GIS analyses, and mapping were done with R, version 3.1 (R Foundation for Statistical Computing; Vienna, Austria).

\section{RESULTS}

\section{Patient Characteristics}

In the seven healthcare networks participating in the pilot Pain SCAN-ECHO program, we identified 371,646 patients with chronic pain using VA primary care who met study criteria (Figure 1). Among these patients, 6.7 percent $(n=25,168)$ were part of the patient panels of primary care providers who presented $\geq 1$ case at Pain SCAN-ECHO sessions, and 17.3 percent $(n=$ 64,394 ) had an in-person specialty pain care clinic visit. The median distance from a patients' home to the nearest pain specialty care clinic was 17.04 miles (interquartile range $=7.24-39.02$ ). Overall, 81 percent of patients lived within 50 miles of a pain specialty clinic. The population was 22.5 percent rural (Table 1).

\section{Healthcare Networks}

There were seven healthcare networks with multiple U.S. states per network that contained a Pain SCANECHO program. The distribution of patients' median distance to specialty pain care showed large network variation, with median distance ranging from 10.6 to 28.7 miles, and the proportion of patients living within 50 miles of pain specialty care ranging from 62 to 96 percent (Table 1). There was also significant network variation in both the proportion of chronic pain patients seen in a specialty pain care clinic (ranging from $10.5 \%$ to $21.5 \%$ ) and the proportion cared for by a provider participating in Pain SCAN-ECHO (ranging 1.8\% to 19.3\%) (Table 1).

\section{Spatial Distribution}

Using GIS mapping in a single network as an example, the density of Veterans with chronic pain mirrored the general VHA population, with a higher number of

Table 1.

Characteristics of Veterans Health Administration (VHA) networks participating in Pain Specialty Care Access Network-Extension for Community Healthcare Outcomes (SCAN-ECHO).

\begin{tabular}{|c|c|c|c|c|c|c|}
\hline $\begin{array}{c}\text { VHA } \\
\text { Healthcare } \\
\text { Network }\end{array}$ & $\begin{array}{c}\text { Total No. } \\
\text { Veterans with } \\
\text { Chronic Pain, } n\end{array}$ & $\begin{array}{l}\text { Total No. SCAN- } \\
\text { ECHO-Exposed } \\
\text { Patients, } n(\%)\end{array}$ & $\begin{array}{c}\text { Total No. Veterans } \\
\text { Seen at In-Person } \\
\text { Pain Specialty Care, } \\
n(\%)\end{array}$ & $\begin{array}{c}\text { Proportion of } \\
\text { Veterans with } \\
\text { Chronic Pain } \\
\text { Living }<50 \text { miles } \\
\text { from Pain } \\
\text { Specialty Care }\end{array}$ & $\begin{array}{c}\text { Distance from } \\
\text { Veteran Home to Pain } \\
\text { Specialty Care, } \\
\text { Median (IQR) (miles) }\end{array}$ & $\begin{array}{c}\text { Proportion } \\
\text { of Veterans } \\
\text { Classified as } \\
\text { Rural }\end{array}$ \\
\hline All & 371,646 & $25,168(6.7)$ & $64,394(17.3)$ & 0.81 & $17.04(7.24-39.02)$ & 0.23 \\
\hline 1 & 45,744 & 3,892 (8.5) & 9,116 (19.9) & 0.88 & 18.63 (8.94-33.48) & 0.27 \\
\hline 2 & 47,391 & 849 (1.8) & 8,021 (16.9) & 0.89 & 17.95 (6.67-33.55) & 0.23 \\
\hline 3 & 64,682 & 2,158 (3.3) & 8,994 (13.9) & 0.80 & $27.34(10.42-46.55)$ & 0.34 \\
\hline 4 & 52,347 & 10,082 (19.3) & $10,977(21.0)$ & 0.96 & $10.64(4.82-22.73)$ & 0.22 \\
\hline 5 & 50,540 & $2,033(4.0)$ & 7,826 (15.5) & 0.62 & 20.17 (7.57-91.61) & 0.24 \\
\hline 6 & 39,705 & 2,723 (6.9) & 4,157 (10.5) & 0.60 & $28.72(6.50-142.57)$ & 0.29 \\
\hline 7 & 71,237 & $3,431(4.8)$ & 15,303 (21.5) & 0.89 & $12.8(7.92-24.36)$ & 0.05 \\
\hline
\end{tabular}


Table 1.

Characteristics of Veterans Health Administration (VHA) networks participating in Pain Specialty Care Access Network-Extension for Community Healthcare Outcomes (SCAN-ECHO).

$\mathrm{IQR}$ = interquartile range, No. = number.

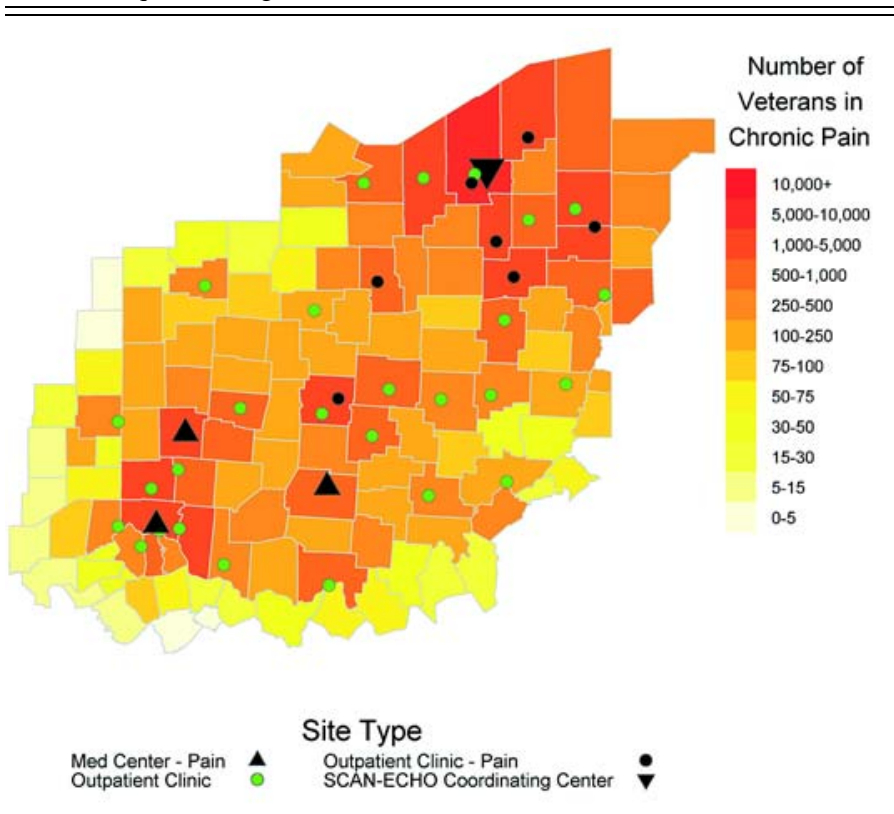

Figure 2.

Geographic distribution of Veterans with chronic pain in sample health network, including Veterans Health Administration medical centers (Med Center) and clinics both with and without pain specialty care available. SCAN-ECHO = Specialty Care Access Network-Extension for Community Healthcare Outcomes.

Veterans living in urban areas around a medical center (Figure 2). Within this network, Veterans living in counties furthest from the coordinating SCAN-ECHO site had a lower probability of access to a provider participating in the Pain SCAN-ECHO program (Figure 3). Conversely, Veterans living closer to the coordinating SCANECHO site were more likely to be treated by a provider who had presented a SCAN-ECHO session. Thus, rural Veterans living further away from the SCAN-ECHO coordinating center had a lower probability of being affected by Pain SCAN-ECHO than rural Veterans living closer to the coordinating center. Veterans living further from specialty pain care sites appeared to be less likely to be seen in-person at a specialty pain clinic (Figure 4).

\section{Association Between Distance and Outcomes}

Across all regions, increasing distance to specialty pain care was associated with significantly lower odds of
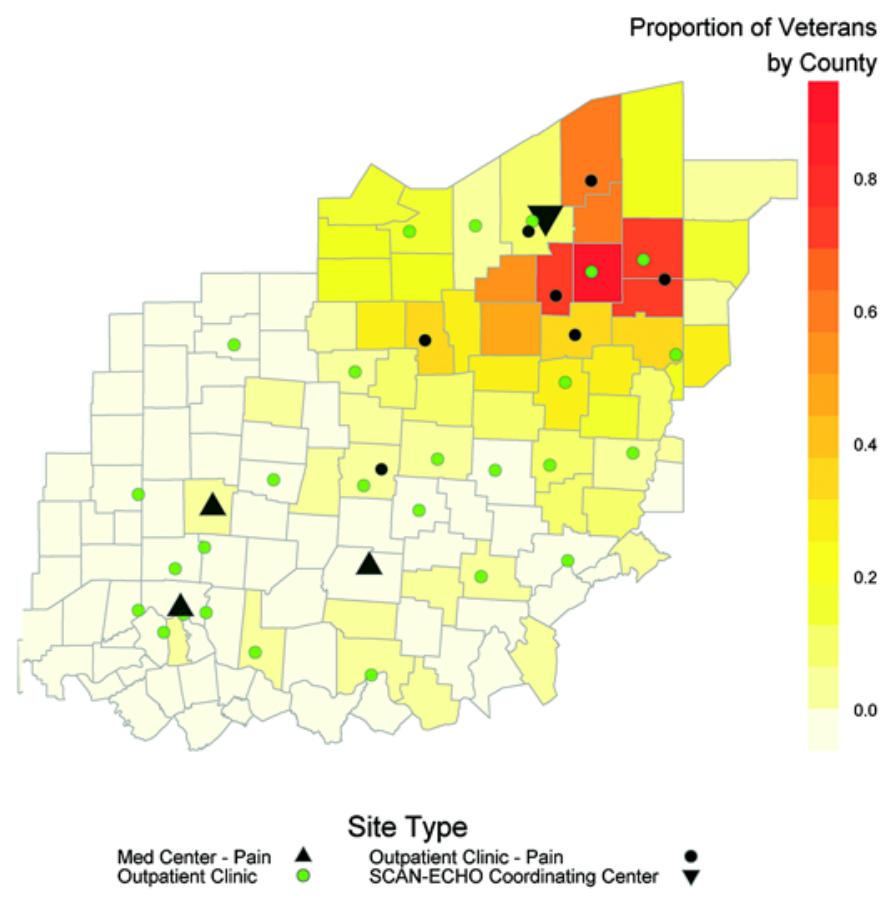

Figure 3.

Geographic distribution of probability of SCAN-ECHO exposure by county for Veterans with chronic pain in sample health network, including Veterans Health Administration medical centers (Med Center) and clinics both with and without pain specialty care. SCAN-ECHO = Specialty Care Access Network-Extension for Community Healthcare Outcomes.

being seen in-person in a specialty pain clinic (Table 2). For every 50-mile increase in Veteran distance from home to specialty pain care, there was a 22 percent lower odds of being seen in person at a specialty pain care clinic (OR $=0.78$ per 50-mile increase, 95\% CI $=0.77-$ $0.79, p<0.001)$. In contrast, for every 50 -mile increase in Veteran distance from home to pain specialty care, there was only a 2 percent lower odds of access to a Pain SCAN-ECHO participating primary care provider $(\mathrm{OR}=$ 0.98 per 50-mile increase, $95 \%$ CI $=0.97-0.99, p=$ 0.01). Logistic regression models stratified by healthcare network revealed large heterogeneity in these results (Table 2). Two of seven regions showed a positive association between distance and Pain SCAN-ECHO pro- 


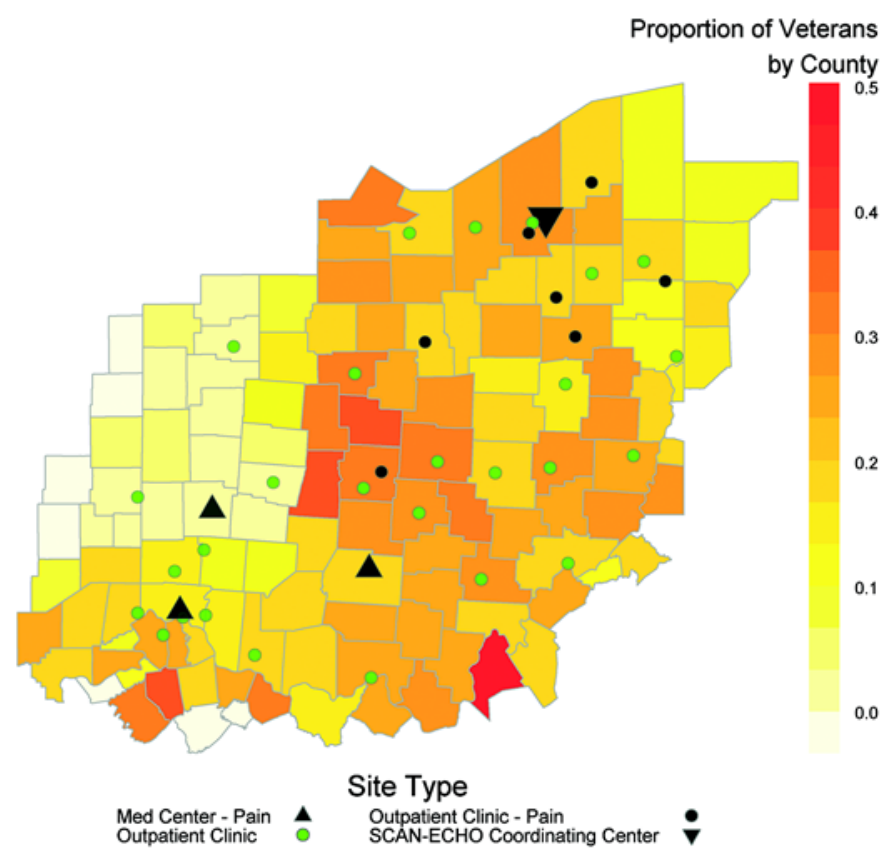

Figure 4.

Geographic distribution of probability of receiving in-person pain specialty care by county for Veterans with chronic pain in sample health network, including Veterans Health Administration medical centers (Med Center) and clinics both with and without pain specialty care. SCAN-ECHO = Specialty Care Access Network-Extension for Community Healthcare Outcomes.

vider participation, indicating Veterans with greater distance to the nearest specialty pain care site had a higher probability of access to a Pain SCAN-ECHO participating provider. Conversely, five of seven regions had a negative association, indicating Veterans living closer to the nearest specialty pain care site had a higher probability of access to a Pain SCAN-ECHO participating provider.

Table 2.

Table of logistic regression model results; odds ratios and 95 percent confidence intervals per 50-mile increase in distance.

\begin{tabular}{ccc}
$\begin{array}{c}\text { Health } \\
\text { Network }\end{array}$ & $\begin{array}{c}\text { Seen In-Person at Pain } \\
\text { Clinic }\end{array}$ & $\begin{array}{c}\text { Touched by Pain } \\
\text { SCAN-ECHO }\end{array}$ \\
\hline All & $0.78(0.77-0.79)$ & $0.98(0.97-1.00)$ \\
1 & $0.96(0.92-1.00)$ & $0.81(0.75-0.86)$ \\
2 & $1.07(1.00-1.11)$ & $0.76(0.63-0.91)$ \\
3 & $0.94(0.90-0.98)$ & $0.80(0.73-0.88)$ \\
4 & $0.84(0.78-0.90)$ & $0.15(0.13-0.16)$ \\
5 & $0.76(0.74-0.77)$ & $1.67(1.62-1.71)$
\end{tabular}

Table 2.

Table of logistic regression model results; odds ratios and 95 percent confidence intervals per 50-mile increase in distance.

$$
\begin{array}{lll}
6 & 0.77(0.75-0.78) & 0.61(0.59-0.64) \\
7 & 1.07(1.04-1.11) & 6.25(5.98-6.52)
\end{array}
$$

Note: Two outcomes were used: Pain Clinic and Pain SCAN-ECHO provider participation. Separate models were fit for all networks. Primary predictor is distance from Veteran home to nearest in-person pain specialty care, with odds ratio presented per 50 mile increase.

SCAN-ECHO $=$ Specialty Care Access Network-Extension for Community Healthcare Outcomes (SCAN-ECHO).

\section{DISCUSSION}

The objective of this study was to evaluate the spatial reach of the VHA's Pain SCAN-ECHO program. We investigated spatial reach, or Veterans' access to a participating primary care provider, in two distinct ways. First, GIS mapping indicated that within a sample network, Pain SCAN-ECHO programs primarily touched Veterans closer to the SCAN-ECHO coordinating center. Rural Veterans living on the other side of the network were not touched by the program, but rural Veterans living closer to the coordinating center were touched. Second, using distance to the nearest in-person pain care as a surrogate for spatial access, we found the probability of Pain SCAN-ECHO provider participation was only mildly associated with distance to specialty pain care in the national cohort, suggesting that the Pain SCAN-ECHO program affected patients with both low and high spatial access to existing specialty pain care. Importantly, we found significant regional variation in these findings. Five of seven networks showed a negative association, indicating they disproportionately affected those patients with better spatial access. The remaining two networks showed a strong positive relationship, indicating Pain SCAN-ECHO disproportionately affected patients with low access to specialty pain care, consistent with the goals of the program. This evaluation is the first study to assess the geographic reach of a technology-based healthcare program in the context of the relevant underlying patient population, current healthcare system resources, and existing access to specialty care.

Prior studies of utilization as a function of spatial access have focused on in-person specialty care [15-19]. Our finding of decreased probability of in-person specialty pain care utilization as distance increases is consistent with those prior studies [15-19]. Among rural patients in North Carolina, increasing distance to care 
was conversely associated with regular and chronic care utilization, although not acute care utilization [19]. A nationwide study of Veterans eligible for liver transplant showed increasing distance to a transplant center was associated with decreasing probability of receiving a liver transplant [18]. Rural Veterans have been shown to have decreased access to hepatitis $C$ specialty care than their urban counterparts [20]. Our findings add to this list by exploring access to technology-based specialty care in addition to in-person specialty care.

GIS techniques have been previously used in evaluation of telemedicine programs. Maps of Project ECHO coordinating sites and mentee sites in New Mexico showed large spatial penetration of mentee sites in New Mexico across different specialty care areas [21]. A nationwide GIS analysis of VHA SCAN-ECHO across all specialties visualized coverage of mentee sites [6]. A regional U.S. telehealth program was evaluated using GIS and demonstrated substantial travel savings associated with telehealth visits compared with hypothetical inperson visits [8].

Several prior studies of Project ECHO programs have examined barriers to implementation of Project ECHO-type programs at the provider and program level [2,22-23]. Providers participating in a Project ECHO program identified the lack of protected time as a main barrier to participation, given their competing clinical duties in high-volume clinics [2,22]. Providers also identified a lack of administrative support as a barrier to participation [2]. Another study noted the main threat to the continuation of their Project ECHO program was financial [23]; the program is funded by grants, but administrative support or reimbursement programs that incentivize these programs are required for the longevity of the programs. These studies all highlight potential reasons why the observed uptake of Pain SCAN-ECHO was variable by region.

Our study has several advantages compared with the existing literature. We included the entire target population (Veterans with chronic pain) of the Pain SCANECHO program. By calculating the distance to in-person specialty pain care resources for all Veterans with chronic pain in a network, we were able to contextualize the spatial penetration of the Pain SCAN-ECHO program against existing in-person specialty pain care utilization. No prior study has used maps to simultaneously visualize the full target patient population, system resource locations, probability of utilization of existing specialty care, and probability of primary care provider participation in the telementoring project. Finally, using patient data from the largest integrated medical system in the United States across seven geographically diverse regions with distinct Pain SCAN ECHO programs, we were able to characterize heterogeneity in implementation across sites.

There are two related but distinct findings that should inform future VHA policy. First, GIS mapping of SCANECHO participation can identify geographic trends with respect to the coordinating site and provide novel insights into program uptake. Our maps suggest that within most participating networks, the Pain SCAN-ECHO programs primarily affected providers and patients who live in relatively close proximity to the coordinating SCAN-ECHO center. In this pilot program, funding mechanisms focused on the coordinating center. A combination of funding and existing intraprofessional relationships are the likely explanations for this finding. Clinics at further distances from the coordinating center may have less administrative support for the program as a result of decreased intraprofessional leadership contact, leading to lower provider participation rates. Future SCAN-ECHO expansion should seek to understand barriers to provider engagement and should specifically target provider engagement in clinics independent of distance to SCANECHO coordinating sites. Administrative support for these programs will be key.

Second, the distribution of Pain SCAN-ECHO provider participation varied widely across the seven participating networks. Overall, we found that SCAN-ECHO provider participation was not associated with distance to specialty pain care, while in-person pain care was negatively associated with distance. However, examining these trends by network showed clear heterogeneity across networks with Pain SCAN-ECHO provider participation positively associated with distance to existing specialty pain care in some networks and negatively associated in others. This heterogeneity may be explained by a combination of variable local implementation strategies and intraprofessional networks as well as variable baseline traits of networks. Prior work has identified administrative support and protected time for participants as a main barrier to participation. Administrative support is likely variable by center (and therefore region), which may contribute to our observed heterogeneity. The distribution of distance to the nearest specialty pain care resources varied considerably by network, which is likely a function of both overall population density and the dis- 
tribution of existing specialty pain care resources in a given network. Given the observed network heterogeneity, evaluation of Pain SCAN-ECHO and other regionally implemented programs should be completed at the network level to guide future implementation.

This analysis provides a framework for targeting the future spread of Pain SCAN-ECHO programs in the VHA as well as other technology-based programs. First, the analytic techniques employed are generalizable beyond the focus of Veterans with chronic pain. GIS techniques hold the potential to identify clusters of patients with low access to in-person specialty care resources and high incidence of disease regardless of disease process. Evaluation of existing technology-based healthcare programs and guidance of future expansion should consider the spatial distribution of the target patient population, the location of existing healthcare system resources, and the location of proposed new healthcare resources. If a primary goal of the program is to extend specialty care access to patients who are currently underserved, an understanding of the current location of patients and resources is critical to finding highvalue targets for expansion of resources. Second, the finding of significant network variation is also informative outside of the VHA. Integrated healthcare systems that cover large and variable geographic areas will almost certainly have regional variation in specialty care availability as well as heterogeneity in implementation of programs in these regions. Region-specific GIS analysis as described here can guide administrators in allocating new specialty care resources.

Interpretation of this study requires recognizing potential limitations. First, any cohort study of administrative data may suffer from confounding due to unmeasured variables. Second, all distance calculations used geodesic ("as the crow flies") distance, which underestimates driving distance. However, prior work has shown a high correlation between geodesic and travel distance [24], especially at higher miles. The primary aim of this study was to contrast distance associations between models and networks, so this potential underestimation should cancel out across comparisons. Third, patientlevel Pain SCAN-ECHO reach was defined based on linking presented patients to their primary care providers. Providers who attended Pain SCAN-ECHO sessions but did not ever present patients were not captured as participating providers, thus we may be undercounting exposed patients. Finally, in-person specialty pain care sites were defined based on the frequency of outpatient pain clinic records. This binary classification represents a nonbinary underlying spectrum of pain care services. It is possible that sites with a small number of pain visits have been misclassified as nonpain care sites. However, only a small percent of sites were on the threshold, and a sample of 15 of these threshold sites that were contacted directly all offered some form of specialty pain care.

\section{CONCLUSIONS}

Among Veterans with chronic pain receiving care in VHA settings, the association between distance to in-person pain specialty care and Pain SCAN-ECHO provider participation was highly variable by region. GIS-based analyses of patient and system resource locations can improve our understanding of program implementation and should inform outreach strategies for technologybased healthcare programs to strategically target Veterans with low access to care.

\section{ACKNOWLEDGMENTS}

\section{Author Contributions:}

Study concept and design: E. P. Carey, J. W. Frank, R. D. Kerns, P. M. Ho, S. R. Kirsh.

Data collection: E. P. Carey.

Data analysis: E. P. Carey.

Data interpretation: E. P. Carey, J. W. Frank, R. D. Kerns, P. M. Ho, S. R. Kirsh.

Drafting of manuscript: E. P. Carey, J. W. Frank.

Critical revision of manuscript for important intellectual content:

E. P. Carey, J. W. Frank, R. D. Kerns, P. M. Ho, S. R. Kirsh.

Financial Disclosures: The authors have declared that no competing interests exist.

Funding/Support: This material was based on work supported by the VA Office of Specialty Care and VA Health Services Research and Development Quality Enhancement Research Initiative and undertaken by the Specialty Care Evaluation Center.

Additional Contributions: This work was presented previously at the AcademyHealth Annual Research Meeting on June 14, 2015, in Minneapolis, Minnesota.

Institutional Review: This was a VHA operational evaluation project that was sponsored by the VA Office of Specialty Care Services/ Specialty Care Transformation. The activities undertaken in the conduct of this project were in support of VHA operational programs and did not constitute research, in whole or in part, in compliance with VHA Handbook 1058.05. Therefore, institutional review board approval was not required. 
Participant Follow-Up: The authors have no plans to notify the study subjects of the publication of this article because of a lack of contact information.

Disclaimer: The views expressed in this article are those of the authors and do not necessarily reflect the position or policy of the VA.

\section{REFERENCES}

1. Katzman JG, Comerci G Jr, Boyle JF, Duhigg D, Shelley B, Olivas C, Daitz B, Carroll C, Som D, Monette R, Kalishman $\mathrm{S}$, Arora S. Innovative telementoring for pain management: Project ECHO pain. J Contin Educ Health Prof. 2014;34(1):68-75. [PMID:24648365] http://dx.doi.org/10.1002/chp.21210

2. Scott JD, Unruh KT, Catlin MC, Merrill JO, Tauben DJ, Rosenblatt R, Buchwald D, Doorenbos A, Towle C, Ramers CB, Spacha DH. Project ECHO: A model for complex, chronic care in the Pacific Northwest region of the United States. J Telemed Telecare. 2012;18(8):481-84.

[PMID:23209269]

http://dx.doi.org/10.1258/jtt.2012.GTH113

3. Arora S, Thornton K, Jenkusky SM, Parish B, Scaletti JV. Project ECHO: Linking university specialists with rural and prison-based clinicians to improve care for people with chronic hepatitis C in New Mexico. Public Health Rep. 2007;122(Suppl 2):74-77. [PMID:17542458]

4. Nassar BS, Vaughan-Sarrazin MS, Jiang L, Reisinger HS, Bonello R, Cram P. Impact of an intensive care unit telemedicine program on patient outcomes in an integrated health care system. JAMA Intern Med. 2014;174(7):1160-67.

[PMID:24819673]

http://dx.doi.org/10.1001/jamainternmed.2014.1503

5. Arora S, Thornton K, Murata G, Deming P, Kalishman S, Dion D, Parish B, Burke T, Pak W, Dunkelberg J, Kistin M, Brown J, Jenkusky S, Komaromy M, Qualls C. Outcomes of treatment for hepatitis $\mathrm{C}$ virus infection by primary care providers. N Engl J Med. 2011;364(23):2199-207.

[PMID:21631316]

http://dx.doi.org/10.1056/NEJMoa1009370

6. Kirsh S, Su GL, Sales A, Jain R. Access to outpatient specialty care: Solutions from an integrated health care system. Am J Med Qual. 2015;30(1):88-90.

[PMID:25024460] http://dx.doi.org/10.1177/1062860614542844

7. Armstrong AW, Kwong MW, Chase EP, Ledo L, Nesbitt TS, Shewry SL. Teledermatology operational considerations, challenges, and benefits: The referring providers' perspective. Telemed J E Health. 2012;18(8):580-84.

[PMID:22881579]

http://dx.doi.org/10.1089/tmj.2011.0241
8. Soares NS, Johnson AO, Patidar N. Geomapping telehealth access to developmental-behavioral pediatrics. Telemed J E Health. 2013;19(8):585-90. [PMID:23756240]

http://dx.doi.org/10.1089/tmj.2012.0226

9. Bramanti A, Bonanno L, Celona A, Bertuccio S, Calisto A, Lanzafame P, Bramanti P. GIS and spatial analysis for costs and services optimization in neurological telemedicine. In: Proceedings of the 2010 Annual International Conference of the IEEE Engineering in Medicine and Biology Society (EMBC); 2010 Aug 31-Sep 4; Buenos Aires, Argentina. New York (NY): IEEE; 2010. p. 2204-7.

10. Clark JD. Chronic pain prevalence and analgesic prescribing in a general medical population. J Pain Symptom Manage. 2002;23(2):131-37. [PMID:11844633] http://dx.doi.org/10.1016/S0885-3924(01)00396-7

11. Veterans Health Administration Office of Rural Health. VHA Office of Rural Health Fact Sheet [Internet]. Washington (DC): Department of Veterans Affairs; 2014 May [cited 2014 Oct 20]. Available from:

http://www.ruralhealth.va.gov/docs/factsheets/ ORH General FactSheet 2014.pdf

12. Dobscha SK, Morasco BJ, Duckart JP, Macey T, Deyo RA. Correlates of prescription opioid initiation and long-term opioid use in veterans with persistent pain. Clin J Pain. 2013;29(2):102-08. [PMID:23269280] http://dx.doi.org/10.1097/AJP.0b013e3182490bdb

13. Department of Veterans Affairs, Veterans Health Administration. Directive VH. 2009-053 Pain management [Internet]. Washington (DC): Department of Veterans Affairs; 2009 Oct [cited 2014 Oct 20]. Available from: http://www.va.gov/painmanagement/docs/ vha09paindirective.pdf

14. West AN, Lee RE, Shambaugh-Miller MD, Bair BD, Mueller KJ, Lilly RS, Kaboli PJ, Hawthorne K. Defining “rural” for veterans' health care planning. J Rural Health. 2010;26(4):301-09. [PMID:21029164] http://dx.doi.org/10.1111/j.1748-0361.2010.00298.x

15. Carr BG, Branas CC, Metlay JP, Sullivan AF, Camargo CA Jr. Access to emergency care in the United States. Ann Emerg Med. 2009;54(2):261-69. [PMID:19201059] http://dx.doi.org/10.1016/j.annemergmed.2008.11.016

16. Hassan A, Pearce NJ, Mathers J, Veugelers PJ, Hirsch GM, Cox JL; Improving Cardiovascular Outcomes in Nova Scotia Investigators. The effect of place of residence on access to invasive cardiac services following acute myocardial infarction. Can J Cardiol. 2009;25(4):207-12.

[PMID:19340343] http://dx.doi.org/10.1016/S0828-282X(09)70062-5

17. Gregory PM, Malka ES, Kostis JB, Wilson AC, Arora JK, Rhoads GG. Impact of geographic proximity to cardiac revascularization services on service utilization. Med Care. 
JRRD, Volume 53, Number 1, 2016

2000;38(1):45-57. [PMID:10630719]

http://dx.doi.org/10.1097/00005650-200001000-00006

18. Goldberg DS, French B, Forde KA, Groeneveld PW, Bittermann T, Backus L, Halpern SD, Kaplan DE. Association of distance from a transplant center with access to waitlist placement, receipt of liver transplantation, and survival among US veterans. JAMA. 2014;311(12):1234-43.

[PMID:24668105]

http://dx.doi.org/10.1001/jama.2014.2520

19. Arcury TA, Gesler WM, Preisser JS, Sherman J, Spencer J, Perin J. The effects of geography and spatial behavior on health care utilization among the residents of a rural region. Health Serv Res. 2005;40(1):135-55. [PMID:15663706] http://dx.doi.org/10.1111/j.1475-6773.2005.00346.x

20. Rongey C, Shen H, Hamilton N, Backus LI, Asch SM, Knight S. Impact of rural residence and health system structure on quality of liver care. PLoS ONE. 2013;8(12): e84826. [PMID:24386420] http://dx.doi.org/10.1371/journal.pone.0084826

21. Arora S, Kalishman S, Thornton K, Dion D, Murata G, Deming P, Parish B, Brown J, Komaromy M, Colleran K, Bankhurst A, Katzman J, Harkins M, Curet L, Cosgrove E, Pak W. Expanding access to hepatitis $\mathrm{C}$ virus treatmentExtension for Community Healthcare Outcomes (ECHO) project: Disruptive innovation in specialty care. Hepatology. 2010;52(3):1124-33. [PMID:20607688] http://dx.doi.org/10.1002/hep.23802

22. Salgia RJ, Mullan PB, McCurdy H, Sales A, Moseley RH, Su GL. The educational impact of the Specialty Care Access Network-Extension of Community Healthcare Outcomes program. Telemed J E Health. 2014;20(11):1004-08.

\section{[PMID:25226452]}

http://dx.doi.org/10.1089/tmj.2013.0302

23. Khatri K, Haddad M, Anderson D. Project ECHO: Replicating a novel model to enhance access to hepatitis $\mathrm{C}$ care in a community health center. J Health Care Poor Underserved. 2013;24(2):850-58. [PMID:23728050]

http://dx.doi.org/10.1353/hpu.2013.0093

24. Haynes R, Jones AP, Sauerzapf V, Zhao H. Validation of travel times to hospital estimated by GIS. Int J Health Geogr. 2006;5:40. [PMID:16984650]

http://dx.doi.org/10.1186/1476-072X-5-40

Submitted for publication October 21, 2014. Accepted in revised form September 11, 2015.

This article and any supplementary material should be cited as follows:

Carey EP, Frank JW, Kerns RD, Ho PM, Kirsh SR. Implementation of telementoring for pain management in Veterans Health Administration: Spatial analysis. J Rehabil Res Dev. 2016;53(1):147-56.

http://dx.doi.org/10.1682/JRRD.2014.10.0247

ORCID: Evan P. Carey, MS: 0000-0001-7963-6818; Robert D. Kerns, PhD: 0000-0001-7609-2771

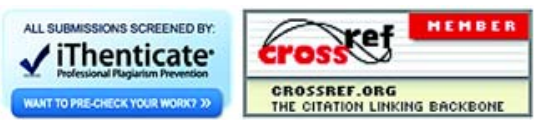

